\title{
SPATIAL MODELS OF BOOLEAN ACTIONS AND GROUPS OF ISOMETRIES
}

\author{
ALEKSANDRA KWIATKOWSKA AND SŁAWOMIR SOLECKI
}

\begin{abstract}
Given a Polish group $G$ of isometries of a locally compact separable metric space, we prove that each measure preserving Boolean action by $G$ has a spatial model or, in other words, has a point realization. This result extends both a classical theorem of Mackey and a recent theorem of Glasner and Weiss, and it covers interesting new examples. In order to prove our result, we give a characterization of Polish groups of isometries of locally compact separable metric spaces which may be of independent interest. The solution to Hilbert's fifth problem plays an important role in establishing this characterization.
\end{abstract}

\section{INTRODUCTION}

Let $(X, \mathcal{B}(X), \mu)$ be a standard Borel space (i.e., there is a Polish topology on $X$ whose family of Borel sets is $\mathcal{B}(X))$ with a Borel probability measure $\mu$ on $\mathcal{B}(X)$. For $B \in \mathcal{B}(X)$, let $[B]_{\mu}$ be the $\mu$-equivalence class of $B$, that is, the set of all $C \in \mathcal{B}(X)$ with $\mu(B \triangle C)=0$. By $\mathcal{B}(X) / \mu$ we denote the Boolean algebra of all $[B]_{\mu}, B \in \mathcal{B}(X)$, with the usual Boolean operations. Let $\operatorname{Aut}(\mu)$ denote the Polish group of all measure preserving automorphisms of $\mathcal{B}(X) / \mu$. We view $\operatorname{Aut}(\mu)$ as a closed subgroup of the orthogonal group $\mathrm{O}\left(L^{2}(\mu)\right)$, where the latter group is equipped with the strong operator topology, by associating with $T \in \operatorname{Aut}(\mu)$ an orthogonal operator $O_{T} \in \mathrm{O}\left(L^{2}(\mu)\right)$ given, with some abuse of notation, by

$$
O_{T}(f)=f \circ T^{-1} \text {. }
$$

(By $L^{2}(\mu)$ we understand the real-valued $L^{2}(\mu)$.)

2000 Mathematics Subject Classification. 03E15, 22A25, 22E15, 22F10, 37A15.

Key words and phrases. Point realizations, Boolean actions, groups of isometries, Hilbert's 5th problem.

Research supported by NSF grant DMS-0700841. 
Let $G$ be a Polish group. Assume we are given a continuous homomorphism $\pi: G \rightarrow \operatorname{Aut}(\mu)$, which we will view as a continuous action of $G$ on $\mathcal{B}(X) / \mu$ :

$$
G \times \mathcal{B}(X) / \mu \ni\left(g,[B]_{\mu}\right) \rightarrow g \cdot[B]_{\mu}=\pi(g)\left([B]_{\mu}\right) \in \mathcal{B}(X) / \mu .
$$

We call such an action a measure preserving Boolean action of $G$ on $\mathcal{B}(X) / \mu$. By a spatial model of such a Boolean action we mean a Borel action $G \times X \rightarrow X$ of $G$ on $X$ such that for each $B \in \mathcal{B}(X)$ and $g \in G$, we have

$$
[g(B)]_{\mu}=g \cdot[B]_{\mu} .
$$

The basic question in this context is: for what Polish groups $G$ each measure preserving Boolean action of $G$ admits a spatial model? By the classical theorem of Mackey [9] every measure preserving Boolean action of a locally compact Polish group admits a spatial model. By the theorem of Glasner-Weiss [8, Theorem 2.3] every measure preserving Boolean action of a closed subgroup of permutations of natural numbers admits a spatial model. Groups in both these classes are Polish groups of isometries of locally compact separable metric spaces: a locally compact Polish group acts faithfully on itself by left translations preserving a left-invariant metric; a closed subgroup of permutations of $\mathbb{N}$ has a natural faithful action on $\mathbb{N}$ preserving the metric assigning distance 1 to each pair of distinct points in $\mathbb{N}$.

In Theorem 1.1, proved in Section 3, we extend the two results above to all Polish groups of isometries of locally compact separable metric spaces. Here is a precise definition of this class of groups. Given a separable metric space $(X, d)$, by $\operatorname{Iso}(X)$ we understand the group of all isometries of $(X, d)$ with composition as group operation and with the topology of pointwise convergence. The group Iso $(X)$ is a separable metrizable topological group. We say that a topological group $G$ is a group of isometries of $X$ if there exists an isomorphism that is also a homeomorphism between $G$ and a subgroup of Iso $(X)$. Of primary interest to us will be Polish groups $G$ that are groups of isometries of locally compact separable metric spaces $X$. The class of Polish groups of isometries of locally compact separable metric spaces admits an alternative description due to Gao and Kechris [6] as the class of all 
closed subgroups of countable products of groups of the form

$$
S_{\infty} \ltimes H^{\mathbb{N}}
$$

where $S_{\infty}$ is the group of all permutations of $\mathbb{N}, H$ is a locally compact Polish group, and, in forming the semidirect product, we view $S_{\infty}$ as acting on $H^{\mathbb{N}}$ by automorphisms permuting the coordinates. In particular, apart from the two subclasses mentioned above and considered by Mackey and Glasner and Weiss, i.e., locally compact Polish groups and closed subgroups of $S_{\infty}$, the class of Polish groups of isometries of locally compact separable metric spaces contains also, for example, closed subgroups of countable products of locally compact Polish groups. For more information on groups of isometries we refer the reader to Section 2.

Theorem 1.1. Let $G$ be a Polish group of isometries of a locally compact separable metric space. Then each measure preserving Boolean action of $G$ has a spatial model.

As proved by Becker [2] and by Glasner, Tsirelson, and Weiss [7] (see also [8]), not all Polish groups fulfill the conclusion of the theorem above. To give one simple example, recall that it is shown in [2] that this is the case for the Abelian Polish group $A$ of measure equivalence classes of Lebesgue measurable subsets of the interval $[0,1]$. The group operation on $A$ is the symmetric difference and the group topology on it is induced by the metric giving the distance between $a, b \in A$ as the Lebesgue measure of the symmetric difference of $a$ and $b$. A wealth of information on actions of Polish groups without spatial models is given by Glasner, Tsirelson, and Weiss in [7] and [8]. We will use the theory developed in these articles in the proof of Theorem 1.1, see Section 3.

The reader may consult [4] for examples of non-Polish groups (countable inductive limits of locally compact groups; such limits, if Polish, are locally compact) for which each measure preserving Boolean action admits a spatial model.

The following characterization of Polish groups of isometries of locally compact separable metric spaces will be crucial in proving Theorem 1.1 and is of independent interest. Recall that if $H$ is a subgroup of a group $G$, 
$N(H)$ stands for the normalizer of $H$, that is,

$$
N(H)=\left\{g \in G: g H g^{-1}=H\right\} .
$$

Theorem 1.2. Let $G$ be a Polish group. Then $G$ is a group of isometries of a locally compact separable metric space if and only if each neighborhood of 1 contains a closed subgroup $H$ such that the space $G / H$ is locally compact and $N(H)$ is open.

The proof of Theorem 1.2, given in Section 2, uses the work of Gao and Kechris [6] on isometry groups of locally compact separable metric spaces mentioned above. The main technical difficulty in our proof of Theorem 1.2 is showing that the property stated in that theorem is preserved under taking closed subgroups; see Subsection 2.1. Curiously, the argument establishing this preservation property uses the solution of Hilbert's fifth problem.

As a by-product of Theorem 1.2 we obtain the following corollary proved in Subsection 2.3.

Corollary 1.3. Let $G$ be a Polish group of isometries of a locally compact separable metric space, and let $N$ be a closed normal subgroup of $G$. Then $G / N$ is also a Polish group of isometries of a locally compact separable metric space. In other words, the class of Polish groups of isometries of locally compact separable metric spaces is closed under taking images of continuous homomorphisms onto Polish groups.

We will prove Theorem 1.2 first as this result is used in our proof of Theorem 1.1.

\section{Groups of isometries And Proofs of Theorem 1.2 And \\ Corollary 1.3}

Viewing Polish groups as isometry groups of metric spaces provides a natural stratification of the class of all Polish groups. The starting point here is the observation that if $X$ is a Polish metric space, then $\operatorname{Iso}(X)$ is a Polish group. Furthermore, the following relevant results are known:

(i) (Uspenskij [13]) $G$ is a Polish group of isometries of a Polish metric space if and only if $G$ is a Polish group; 
(ii) (Gao-Kechris [6, Theorem 6.3]) $G$ is a Polish group of isometries of a locally compact Polish metric space, or equivalently a locally compact separable metric space, if and only if $G$ is a closed subgroup of a group of the form

$$
\prod_{n \in \mathbb{N}} S_{\infty} \ltimes H_{n}^{\mathbb{N}}
$$

where $S_{\infty}$ is the group of all permutations of $\mathbb{N}$, each $H_{n}$ is a locally compact second countable group, and the semidirect product is formed by viewing each $\sigma \in S_{\infty}$ as an automorphism of $H_{n}^{\mathbb{N}}$ which acts on $h \in H_{n}^{\mathbb{N}}$ by returning $\sigma(h) \in H_{n}^{\mathbb{N}}$ given by

$$
\sigma(h)(i)=h\left(\sigma^{-1}(i)\right)
$$

(iii) (folklore) $G$ is a Polish group of isometries of a proper metric space, that is, a metric space in which all balls are compact, if and only if $G$ is a locally compact second countable group;

(iv) (folklore) $G$ is a Polish group of isometries of a compact metric space if and only if $G$ is a compact second countable group.

Moreover, in the conditions on the left hand side in each of the points above, one can replace the phrase "Polish group" by "closed group." Also, the conditions on the left hand side in each of the points above can be replaced by the condition that $G$ be isomorphic to the whole group $\operatorname{Iso}(X)$ in (i) for a Polish metric space $X$ [6, Theorem 3.1(i)], in (ii) for a locally compact Polish metric space $X$ [6, Theorem 6.3], in (iii) for a proper metric space $X$ [10, Theorem 2.1], and in (iv) for a compact metric space $X$ [11], respectively.

Point (ii) above will be used in the sequel. It provides an approach to Polish groups of isometries of locally compact separable metric spaces by building them from simpler components. The least explicit step in this approach is that of taking closed subgroups and this is where one of the main technical difficulties will appear.

2.1. Direction $\Leftarrow$ of Theorem 1.2. We state explicitly the property from Theorem 1.2:

(*) for every open neighborhood of identity $U \subseteq G$ there is a closed subgroup $H<G$ such that $H \subseteq U, N(H)$ is open, and $G / H$ is locally compact. 
We will be later referring to this property of $G$ as property $(*)$.

In Proposition 2.1 we collect some well known properties of Lie groups that will be used. Important to us will be the notion of dimension of a Lie group, which can be understood as the linear dimension of its Lie algebra or, equivalently, as the dimension of the underlying manifold.

Proposition 2.1. $\quad$ (i) Connected components of a Lie group are open and the connected component of the identity is a Lie group.

(ii) If $M$ is a Lie group and $N$ a closed subgroup of $M$, then $N$ is a Lie group; if, additionally, $N$ is normal, then $M / N$ is a Lie group.

(iii) Let $M, N$ be Lie groups and let $f: M \rightarrow N$ be a continuous homomorphism. If $f$ is injective, then $\operatorname{dim}(M) \leq \operatorname{dim}(N)$; if $f$ is surjective, then $\operatorname{dim}(M) \geq \operatorname{dim}(N)$.

(iv) Let $M, N$ be Lie groups with $\operatorname{dim}(M)=\operatorname{dim}(N)$ and with $N$ connected. If $f: M \rightarrow N$ is a continuous injective homomorphism, then $f$ is surjective.

Proof. (i) The first statement is clear from the definition of manifold. The second statement follows from the first one and the general fact that the connected component of the identity of a topological group is a subgroup.

(ii) See [14, Theorem 3.42] for the proof that $\mathrm{N}$ is Lie and [14, Theorem 3.64] for the proof that $\mathrm{M} / \mathrm{N}$ is Lie.

(iii) This point follows from [14, Theorem 3.32].

(iv) By [14, Theorem 3.32], $f(M)$ is an open, so closed and open, subgroup of $N$. Since $N$ is connected, $f(M)=N$.

We say that a locally compact Polish group $G$ is Lie projective if for every open $U \subseteq G$ there is a compact normal subgroup $K$ of $G$ such that $K \subseteq U$ and $G / K$ is a Lie group. We will use the following deep theorem about locally compact groups.

Theorem 2.2. [12, Theorem 4.6, p.175, Lemma 2.3.1, p.54] Suppose that $G$ is a locally compact group. Then there is an open subgroup $G^{\prime}<G$ that is Lie projective.

Lemma 2.3. Each closed subgroup of a Polish group with property $(*)$ has property $(*)$. 
Proof. First we show that if a Polish group $G_{0}$ has property $(*)$, then it has the following stronger version of that property:

each neighborhood of 1 contains a closed group $H$ such that $N(H)$ is open and $N(H) / H$ is a Lie group.

To prove this property let $U \ni 1$ be open. Let $V \ni 1$ be open such that

$$
V^{2} \subseteq U
$$

By property $(*)$ there exists a closed group

$$
H_{0} \subseteq V
$$

such that $N\left(H_{0}\right)$ is open and $N\left(H_{0}\right) / H_{0}$ is locally compact. By Theorem 2.2 there exists an open subgroup $N$ of $N\left(H_{0}\right)$ such that $H_{0}<N$ and $N / H_{0}$ is Lie projective. We can assume without loss of generality that $V \subseteq N$. Let $\pi: N\left(H_{0}\right) \rightarrow N\left(H_{0}\right) / H_{0}$ be the quotient homomorphism. Then $\pi(V)$ is open. Take

$$
K \subseteq \pi(V)
$$

a compact normal subgroup of $N / H_{0}$ such that $\left(N / H_{0}\right) / K$ is Lie. Let

$$
H=\pi^{-1}(K)
$$

By (1), (2), (3), $H \subseteq U$. Since $K$ is normal in $N / H_{0}, H$ is normal in $N$, and so we have $N(H) \supseteq N$, implying that $N(H)$ is open. Finally, since we have the homeomorphism

$$
N(H) / H=N(H) / \pi^{-1}(K) \cong\left(N(H) / H_{0}\right) / K,
$$

an open subgroup of $N(H) / H$ is isomorphic to the Lie group $\left(N / H_{0}\right) / K$. Thus, $N(H) / H$ is Lie.

Let a Polish group $G_{0}$ with property $(*)$ be given. We can assume that $G_{0}$ has the stronger version of property $(*)$ stated above. Fix closed subgroups $H_{n}<G_{0}$ and open subgroups $M_{n}<G_{0}, n \in \mathbb{N}$, so that $H_{n} \subseteq M_{n}$ and $H_{n}$ is normal in $M_{n}, M_{n} / H_{n}$ is a Lie group, and each open neighborhood of 1 in $G_{0}$ contains $H_{n}$ for all but finitely many $n$. We assume, as we can, that $M_{n+1} \subseteq M_{n}$. Let $\pi_{n}$ be the product of the natural quotient functions $G_{0} \rightarrow G_{0} / H_{i}$

$$
\pi_{n}: G_{0} \rightarrow \prod_{i \leq n} G_{0} / H_{i}
$$


and let $\pi_{n, N}, n \leq N$, be the projection

$$
\pi_{n, N}: \prod_{i \leq N} G_{0} / H_{i} \rightarrow \prod_{i \leq n} G_{0} / H_{i} .
$$

Note that for $N \geq n$

$$
\pi_{n, N} \circ \pi_{N}=\pi_{n}
$$

Let $L_{n}=\prod_{i \leq n} M_{i} / H_{i}$. We point out that $L_{n}$ is a Lie group and that

$$
\pi_{n}\left\lceilM _ { n } : M _ { n } \rightarrow L _ { n } \text { and } \pi _ { n , N } \left\lceil L_{N}: L_{N} \rightarrow L_{n} .\right.\right.
$$

are continuous group homomorphisms.

Let $G<G_{0}$ be closed. We will show that $G$ has property (*). Fix an open neighborhood of 1 in $G$. Find $n$ such that $G \cap H_{n}$ is included in that neighborhood. Note that the normalizer in $G$ of $G \cap \bigcap_{i \leq n} H_{i}$ includes $G \cap M_{n}$ and is, therefore, open in $G$. Thus, to prove that $G$ has property (*), it will suffice to show that for each $n, G /\left(G \cap \bigcap_{i \leq n} H_{i}\right)$ is locally compact.

By Proposition 2.1(ii), the closure in $L_{N}$ of the subgroup $\pi_{N}\left(G \cap M_{N}\right)$ is a Lie group, and, by Proposition 2.1(i), the connected component of the identity of this closure is a Lie group as well. So if we let

$$
A_{N}=\text { the connected component of } 1 \text { of } \overline{\pi_{N}\left(G \cap M_{N}\right)},
$$

then $A_{N}$ is a Lie group. For $n \leq N$, let

$$
B_{n, N}=\operatorname{ker}\left(\pi_{n, N}\left\lceil A_{N}\right) .\right.
$$

Note that since $M_{N+1} \subseteq M_{N}, \pi_{N, N+1}\left(A_{N+1}\right)$ is a connected subgroup of $\overline{\pi_{N}\left(G \cap M_{N}\right)}$, and therefore we have

$$
\pi_{N, N+1}\left(A_{N+1}\right) \subseteq A_{N} .
$$

For $N \geq n, A_{N} / B_{n, N}$ is a Lie group by Proposition 2.1(ii). For these groups we have the following claim.

Claim 1. For every $n$ there is $i_{n} \geq n$ such that for $N \geq i_{n}$

$$
\operatorname{dim}\left(A_{i_{n}} / B_{n, i_{n}}\right)=\operatorname{dim}\left(A_{N} / B_{n, N}\right) .
$$

Proof. Let $N \geq n$. Inclusion (4) induces an injective continuous homomorphism

$$
A_{N+1} /\left(\pi_{N, N+1}^{-1}\left(B_{n, N}\right) \cap A_{N+1}\right) \rightarrow A_{N} / B_{n, N} .
$$


It follows by Proposition 2.1(iii) that

$$
\operatorname{dim}\left(A_{N+1} /\left(\pi_{N, N+1}^{-1}\left(B_{n, N}\right) \cap A_{N+1}\right)\right) \leq \operatorname{dim}\left(A_{N} / B_{n, N}\right) .
$$

Note however that

$$
\pi_{N, N+1}^{-1}\left(B_{n, N}\right) \cap A_{N+1} \subseteq B_{n, N+1},
$$

and, therefore, there is a surjective continuous homomorphism

$$
A_{N+1} /\left(\pi_{N, N+1}^{-1}\left(B_{n, N}\right) \cap A_{N+1}\right) \rightarrow A_{N+1} / B_{n, N+1},
$$

so by Proposition 2.1(iii) we have

$$
\operatorname{dim}\left(A_{N+1} / B_{n, N+1}\right) \leq \operatorname{dim}\left(A_{N+1} /\left(\pi_{N, N+1}^{-1}\left(B_{n, N}\right) \cap A_{N+1}\right)\right) .
$$

From this inequality and from (5) we get

$$
\operatorname{dim}\left(A_{N+1} / B_{n, N+1}\right) \leq \operatorname{dim}\left(A_{N} / B_{n, N}\right) .
$$

We conclude that the natural number valued function

$$
N \rightarrow \operatorname{dim}\left(A_{N} / B_{n, N}\right)
$$

defined for $N \geq n$, is non-increasing, and the conclusion of the claim follows.

For $n \in \mathbb{N}, i_{n} \geq n$ will denote the natural number from Claim 1 .

Claim 2. Let $n \in \mathbb{N}$. For $N \geq i_{n}$,

$$
\pi_{n, N+1}\left(A_{N+1}\right)=\pi_{n, N}\left(A_{N}\right) .
$$

Proof. The homomorphisms $\pi_{n, N} \uparrow A_{N}$ and $\pi_{n, N+1} \uparrow A_{N+1}$ induce injective continuous homomorphisms

$$
\widehat{\pi}_{n, N}: A_{N} / B_{n, N} \rightarrow L_{n} \text { and } \widehat{\pi}_{n, N+1}: A_{N+1} / B_{n, N+1} \rightarrow L_{n} .
$$

Furthermore, from (4), we see that

$$
\begin{aligned}
\widehat{\pi}_{n, N+1}\left(A_{N+1} / B_{n, N+1}\right) & =\pi_{n, N+1}\left(A_{N+1}\right) \\
& \subseteq \pi_{n, N}\left(A_{N}\right)=\widehat{\pi}_{n, N}\left(A_{N} / B_{n, N}\right) .
\end{aligned}
$$

Note that by Claim 1

$$
\operatorname{dim}\left(A_{N+1} / B_{n, N+1}\right)=\operatorname{dim}\left(A_{N} / B_{n, N}\right)
$$


and that $A_{N} / B_{n, N}$ is connected, as $A_{N}$ is. Now since $\widehat{\pi}_{n, N}$ and $\widehat{\pi}_{n, N+1}$ are injective, by (6), we can consider the injective homomorphism

$$
\left(\widehat{\pi}_{n, N}\right)^{-1} \circ \widehat{\pi}_{n, N+1}: A_{N+1} / B_{n, N+1} \rightarrow A_{N} / B_{n, N} .
$$

Since this homomorphism is Borel, it is continuous, and, by what was said above, Proposition 2.1(iv) implies that it is surjective. From this assertion and from (6), the conclusion of the claim follows immediately.

By Claim $2, \pi_{n, N}\left(A_{N}\right)$ does not depend on $N$ as long as $N \geq i_{n}$. Put

$$
C_{n}=\pi_{n, N}\left(A_{N}\right)
$$

for any $N \geq i_{n}$.

Claim 3. For every $n$,

$$
C_{n} \subseteq \pi_{n}\left(G \cap \bigcap_{i} M_{i}\right)
$$

Proof. Note first that for each $n, \pi_{n, n+1}\left(C_{n+1}\right)=C_{n}$. This is because, for $N \geq i_{n}, i_{n+1}$,

$$
\begin{aligned}
C_{n} & =\pi_{n, N}\left(A_{N}\right)=\pi_{n, n+1}\left(\pi_{n+1, N}\left(A_{N}\right)\right) \\
& =\pi_{n, n+1}\left(C_{n+1}\right) .
\end{aligned}
$$

We will use the following general observation concerning Polish groups, see [5, Corollary 2.2.2]. Let $d_{l}$ be a compatible left-invariant metric on $G_{0}$, and let $d_{r}$ be the right-invariant metric on $G_{0}$ given by $d_{r}(x, y)=d_{l}\left(x^{-1}, y^{-1}\right)$. Then the metric $d$ defined by

$$
d=d_{l}+d_{r}
$$

is a complete metric on $G_{0}$. We will also need the following definition. For each $i \in \mathbb{N}$ and $g_{1}, g_{2} \in M_{i}$, let $\rho_{i}$ be given by the formula

$$
\begin{aligned}
\rho_{i}\left(g_{1} H_{i}, g_{2} H_{i}\right)= & \inf \left\{d_{l}\left(g_{1} h_{1}, g_{2} h_{2}\right): h_{1}, h_{2} \in H_{i}\right\} \\
& +\inf \left\{d_{r}\left(g_{1} h_{1}, g_{2} h_{2}\right): h_{1}, h_{2} \in H_{i}\right\} .
\end{aligned}
$$

Since $H_{i}$ is a normal subgroup of $M_{i}$, by [5, Lemma 2.2.8], $\rho_{i}$ is a metric on $M_{i} / H_{i}$ inducing the quotient topology. 
Fix $n_{0}$. Let $y_{0}$ be an arbitrary element of $C_{n_{0}}$. We will show that $y_{0} \in$ $\pi_{n_{0}}\left(G \cap \bigcap_{i} M_{i}\right)$. Using (7), we can recursively pick $c_{n} \in M_{n} / H_{n}$ so that for each $n$ we have

$$
\left(c_{0}, \ldots, c_{n}\right) \in C_{n} \text { and }\left(c_{0}, \ldots, c_{n_{0}}\right)=y_{0} .
$$

By definition of $C_{n}$ and $A_{N}$ we have

$$
C_{n} \subseteq \pi_{n, N}\left(\overline{\pi_{N}\left(G \cap M_{N}\right)}\right) \subseteq \overline{\pi_{n}\left(G \cap M_{n}\right)},
$$

where $N \geq i_{n}$ is arbitrary and where the closure is taken in $L_{n}$. Using this observation and (8), we can pick recursively on $n$ a sequence $g_{n} \in G \cap M_{n}$, $n \in \mathbb{N}$, so that $\pi_{n}\left(g_{n}\right)=\left(g_{n} H_{0}, \ldots, g_{n} H_{n}\right)$ is as close to $\left(c_{0}, \ldots, c_{n}\right)$ as we wish, say, we wish that for all $i \leq n$

$$
\rho_{i}\left(g_{n} H_{i}, c_{i}\right)<\frac{1}{n+1} .
$$

Consider now an arbitrary left coset $g H_{i}$ of $H_{i}$ in $M_{i}$. Since $g \in M_{i}$, we have $g H_{i}=H_{i} g$. Thus, the $d_{l}$-diameter of $g H_{i}$ and the $d_{r}$-diameter of $g H_{i}$ are equal to the $d_{l^{-}}$and the $d_{r^{-}}$-diameters of $H_{i}$, respectively. From this observation, from the definition of $\rho_{i}$, and from (9), it follows that for $i \leq n$

$$
\begin{aligned}
d_{l}\left(g_{i}, g_{n}\right) & \leq d_{l} \text {-diam }\left(g_{i} H_{i}\right)+\frac{1}{i+1}+d_{l}-\operatorname{diam}\left(c_{i}\right)+\frac{1}{n+1}+d_{l}-\operatorname{diam}\left(g_{n} H_{i}\right) \\
& \leq \frac{2}{i+1}+3 \cdot d-\operatorname{diam}\left(H_{i}\right),
\end{aligned}
$$

and similarly

$$
d_{r}\left(g_{i}, g_{n}\right) \leq \frac{2}{i+1}+3 \cdot d-\operatorname{diam}\left(H_{i}\right) .
$$

Thus, for $i \leq n$ we get that

$$
d\left(g_{i}, g_{n}\right) \leq \frac{4}{i+1}+6 \cdot d-\operatorname{diam}\left(H_{i}\right),
$$

and therefore the sequence $\left(g_{i}\right)$ is $d$-Cauchy. Since $d$ is complete, $\left(g_{i}\right)$ converges to some $g_{\infty}$. Since $M_{i+1} \subseteq M_{i}$ and since each $M_{i}$ and $G$ are closed, we see that

$$
g_{\infty} \in G \cap \bigcap_{i} M_{i} .
$$

Furthermore, from (9) we see that for each $n$

$$
\pi_{n}\left(g_{\infty}\right)=\left(c_{0}, \ldots, c_{n}\right),
$$

in particular, by (8), we have $\pi_{n_{0}}\left(g_{\infty}\right)=y_{0}$, as required. 
Now we are ready to finish the proof, that is, show that for every $n$,

$$
G /\left(G \cap \bigcap_{i \leq n} H_{i}\right)
$$

is locally compact. We will apply the following fact that is easy to prove using the Baire category theorem: if a homogeneous Polish space contains an open non-empty subset that is $\sigma$-compact, then the space is itself locally compact. (By a homogeneous space we mean a space in which for each pair of points $x, y$ there is a homeomorphism of the space onto itself mapping $x$ to $y$.) Now fix $n$ and note that $G /\left(G \cap \bigcap_{i \leq n} H_{i}\right)$ is a Polish space with its natural quotient topology. It is homogeneous and it contains the Polish group

$$
\left(G \cap M_{n}\right) /\left(G \cap \bigcap_{i \leq n} H_{i}\right)
$$

as an open subset. Thus, it suffices to show that this last group is $\sigma$-compact.

Take $N \geq i_{n}$. Consider the following commutative diagram. The functions in the diagram are defined below it.

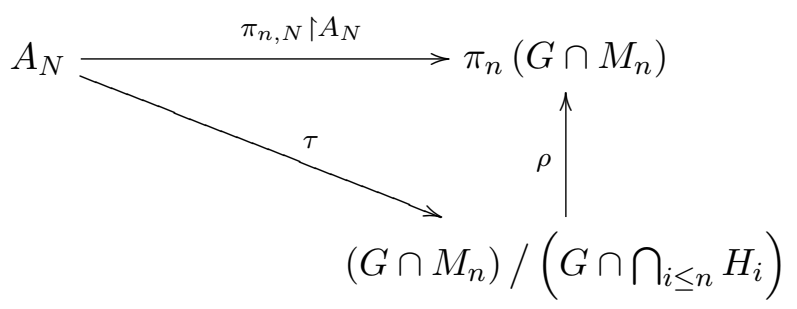

The range of the continuous homomorphism $\pi_{n, N} \uparrow A_{N}$ is included in the group $\pi_{n}\left(G \cap M_{n}\right)$ by Claim 3. The function $\rho$ is defined as follows. In general, given groups $M, N \triangleleft M, N_{0}, \ldots, N_{n} \triangleleft M$ and $K<M$, there exist natural injective homomorphisms

$$
K /(K \cap N) \rightarrow M / N \text { and } M / \bigcap_{i \leq n} N_{i} \rightarrow \prod_{i \leq n} M / N_{i}
$$

From these general principles, we get two continuous injective homomorphisms

$$
\left(G \cap M_{n}\right) /\left(G \cap \bigcap_{i \leq n} H_{i}\right) \rightarrow M_{n} / \bigcap_{i \leq n} H_{i}
$$


and

$$
M_{n} / \bigcap_{i \leq n} H_{i} \rightarrow \prod_{i \leq n} M_{i} / H_{i}=L_{n}
$$

and we let $\rho$ be their compositions. Clearly $\rho$ is a continuous injective homomorphism. By tracing the definitions it is easy to see that $\rho$ is onto $\pi_{n}\left(G \cap M_{n}\right)$; thus, $\rho$ is a continuous isomorphism. We define $\tau$ by

$$
\tau=(\rho)^{-1} \circ \pi_{n, N} .
$$

The function $\tau$ is a homomorphism and it is Borel, since $\rho^{-1}$ is Borel being the inverse of a continuous injection. Since $\tau$ maps a Polish group $A_{N}$ into a second countable group, it is a continuous homomorphism.

Since $A_{N}$ is locally compact, it is $\sigma$-compact, and, therefore, so is $\tau\left(A_{N}\right)$ by continuity of $\tau$. Thus, it suffices to show that $\tau\left(A_{N}\right)$ has countable index in

$$
\left(G \cap M_{n}\right) /\left(G \cap \bigcap_{i \leq n} H_{i}\right)
$$

Since $\rho$ is an isomorphism, it follows from the diagram that it is enough to prove that $\pi_{n, N}\left(A_{N}\right)$ has countable index in $\pi_{n}\left(G \cap M_{n}\right)$.

To prove this assertion note that from the definition of $A_{N}$ and from Proposition 2.1(i), $A_{N}$ is a non-empty relatively open subset of

$$
\overline{\pi_{N}\left(G \cap M_{N}\right)},
$$

which allows us to pick $g_{j} \in G \cap M_{N}, j \in \mathbb{N}$, so that

$$
\bigcup_{j} \pi_{N}\left(g_{j}\right) A_{N}=\overline{\pi_{N}\left(G \cap M_{N}\right)} .
$$

Applying $\pi_{n, N}$ to both sides of the equality above, removing the closure operation, and using $\pi_{n, N} \circ \pi_{N}=\pi_{n}$ and the fact that $\pi_{n, N}$ restricted to the group $L_{N}$ is a homomorphism, we obtain

$$
\bigcup_{j} \pi_{n}\left(g_{j}\right) \pi_{n, N}\left(A_{N}\right) \supseteq \pi_{n}\left(G \cap M_{N}\right)
$$

with $\pi_{n}\left(g_{j}\right) \in \pi_{n}\left(G \cap M_{n}\right)$. Thus, $\pi_{n, N}\left(A_{N}\right)$ will have countable index in $\pi_{n}\left(G \cap M_{n}\right)$ as soon as we show that the index of $\pi_{n}\left(G \cap M_{N}\right)$ in $\pi_{n}\left(G \cap M_{n}\right)$ is countable. But this last assertion is obvious since $\pi_{n} \uparrow\left(G \cap M_{n}\right)$ is a homomorphism and $G \cap M_{N}$ is open and, therefore, of countable index in $G \cap M_{n}$. 
Lemma 2.4. A countable product of Polish groups with property (*) has property $(*)$.

Proof. Assume $G_{n}, n \in \mathbb{N}$, are Polish groups with property $(*)$. Fix an open neighborhood of 1 in $\prod_{n} G_{n}$, which we can assume to be of the form

$$
U_{0} \times \cdots \times U_{m} \times \prod_{n>m} G_{n}
$$

Let $H_{i}<G_{i}, i \leq m$, be closed and such that $H_{i} \subseteq U_{i}, G_{i} / H_{i}$ locally compact, and $N\left(H_{i}\right)$ open. Then the subgroup of $\prod_{n} G_{n}$

$$
H=H_{0} \times \cdots \times H_{m} \times \prod_{n>m} G_{n} .
$$

is as required, that is, $H$ is included in the given neighborhood of 1 , the space $\left(\prod_{n} G_{n}\right) / H$ is locally compact, and $N(H)$ is open.

Proof of $\Leftarrow$ in Theorem 1.2. By the theorem of Gao and Kechris [6, Theorem 6.3] stated in the present paper as point (ii) at the beginning of Section 2, and by Lemmas 2.3 and 2.4, it suffices to show that groups $G$ of the form

$$
G=S_{\infty} \ltimes H^{\mathbb{N}}
$$

have property $(*)$, where $H$ is Polish locally compact and $S_{\infty}$ acts by automorphisms on $H^{\mathbb{N}}$ as follows

$$
\sigma(h)(i)=h\left(\sigma^{-1}(i)\right),
$$

where $\sigma \in S_{\infty}$ and $h \in H^{\mathbb{N}}$. To prove this fact, fix an open neighborhood of $1 \in G$, which we can assume to be of the form

$$
\left\{\sigma \in S_{\infty}: \sigma\lceil n=\mathrm{id}\} \times U^{n} \times H^{\mathbb{N} \backslash n}\right.
$$

for some open $1 \in U \subseteq H$ and some $n \in \mathbb{N}$, where $n$ denotes the set $\{0, \ldots, n-1\}$. Let

$$
G_{0}=\left\{\sigma \in S_{\infty}: \sigma\lceil n=\mathrm{id}\} \ltimes\left(\{1\}^{n} \times H^{\mathbb{N} \backslash n}\right) .\right.
$$

Clearly $G_{0}$ is a closed subgroup of $G$ contained in the given neighborhood of 1 . Its normalizer $N\left(G_{0}\right)$ contains

$$
\left\{\sigma \in S_{\infty}: \sigma \mid n=\mathrm{id}\right\} \times H^{\mathbb{N}}
$$

and therefore is open. The quotient $G / G_{0}$ contains an open subset homeomorphic to $H^{n}$, which makes the space $G / G_{0}$ locally compact. 
2.2. Direction $\Rightarrow$ of Theorem 1.2. In this subsection, we present the following proof. Recall the definition of property $(*)$ from the beginning of Subsection 2.1.

Proof of $\Rightarrow$ of Theorem 1.2. Fix $d$, a left-invariant metric on $G$. Without loss of generality $d$ is bounded by 1 . Fix a decreasing sequence $U_{n}, n \in \mathbb{N}$, of open neighborhoods of 1 in $G$ such that $\operatorname{diam}\left(U_{n}\right) \rightarrow 0$ and $\bigcap_{n} U_{n}=\{1\}$. Let $H_{n} \subseteq U_{n}$ be closed subgroups of $G$ as in property (*). Set $M_{n}=N\left(H_{n}\right)$.

Consider $\oplus_{n}\left(G / H_{n}\right)$, the topological direct sum of the spaces $G / H_{n}$. This is a locally compact separable metrizable space. We define $d^{*}$ by letting, for $x, y \in G$,

$$
d^{*}\left(x H_{n}, y H_{n}\right)=\inf \left\{d(x u, y v): u, v \in H_{n}\right\}
$$

and, if $m \neq n$,

$$
d^{*}\left(x H_{n}, y H_{m}\right)=1
$$

We show that $d^{*}$ is a metric on the set $\oplus_{n}\left(G / H_{n}\right)$. Note first that, by [5, Lemma 2.2.8], for each $n, d^{*}$ restricted to $M_{n} / H_{n}$ is a metric. This remains true, by left-invariance of $d$, for the restriction of $d^{*}$ to $g\left(M_{n} / H_{n}\right)$, for each $g \in G$. Now, all the properties of a metric are clearly true of $d^{*}$ except perhaps for the fact that $x H_{n}=y H_{n}$ whenever $d^{*}\left(x H_{n}, y H_{n}\right)=0$. To show this, fix sequences $\left(u_{i}\right)_{i}$ and $\left(v_{i}\right)_{i}$ in $H_{n}$ such that $d\left(x u_{i}, y v_{i}\right) \rightarrow 0$. Then $d\left(v_{i}^{-1} y^{-1} x u_{i}, 1\right) \rightarrow 0$, which allows us to fix $i_{0}$ such that $v_{i_{0}}^{-1} y^{-1} x u_{i_{0}} \in M_{n}$, implying $y^{-1} x \in M_{n}$. Hence $x$ and $y$ are in the same coset of $M_{n}$ in $G$. Therefore $x H_{n}=y H_{n}$ by the remark at the beginning of this argument.

Notice that, by left invariance of $d$, for each $g \in G$ the function

$$
\oplus_{n}\left(G / H_{n}\right) \ni c \rightarrow g c \in \oplus_{n}\left(G / H_{n}\right)
$$

is an isometry with respect to $d^{*}$.

We show that $d^{*}$ is compatible with the quotient topology on $\oplus_{n}\left(G / H_{n}\right)$. It suffices to see that both topologies agree on $G / H_{n}$, for each $n$. We start by showing that $M_{n} / H_{n}$ is open in each of the two topologies. Clearly this set is open in the quotient topology. For the topology induced by $d^{*}$ suppose that

$$
d^{*}\left(x_{i} H_{n}, x H_{n}\right) \rightarrow 0
$$


where $x_{i} \notin M_{n}$ and $x \in G$. Suppose that $x \in M_{n}$. Since $H_{n}$ is normal in $M_{n}$, for some sequences $\left(u_{i}\right)_{i}$ and $\left(v_{i}\right)_{i}$ in $H_{n}$, we have

$$
d\left(x_{i} u_{i}, v_{i} x\right) \rightarrow 0,
$$

i.e., $d\left(v_{i}^{-1} x_{i} u_{i}, x\right) \rightarrow 0$. Note that for each $i, v_{i}^{-1} x_{i} u_{i} \notin M_{n}$. Hence, since $M_{n}$ is open in $G, x \notin M_{n}$, and we get a contradiction.

Since (10) is an isometry with respect to $d^{*}, g\left(M_{n} / H_{n}\right)$, where $g \in G$, is open in $G / H_{n}$ and homeomorphic to $M_{n} / H_{n}$ with respect to each of the two topologies. Thus, it suffices to see that the two topologies coincide on $M_{n} / H_{n}$, which follows immediately from [5, Lemma 2.2.8].

We show now that $f: G \rightarrow \operatorname{Iso}\left(\oplus_{n}\left(G / H_{n}\right)\right)$ given by

$$
f(g)\left(x H_{n}\right)=(g x) H_{n},
$$

for each $n$, is an embedding of Polish groups. Clearly $f$ is a group homomorphism. Next note that $f$ is injective. Indeed, let $g_{1} \neq g_{2}$. Take $n$ such that $g_{2}^{-1} g_{1} \notin H_{n}$. Then $g_{1} H_{n} \neq g_{2} H_{n}$, i.e., $f\left(g_{1}\right)\left(H_{n}\right) \neq f\left(g_{2}\right)\left(H_{n}\right)$.

Finally, we show that $f$ is a topological embedding. First let $g_{i} \rightarrow g$. We want to show that for each $x \in G$ and $n \in \mathbb{N}, d^{*}\left(g_{i} x H_{n}, g x H_{n}\right) \rightarrow 0$. By the definition of $d^{*}$, we have the inequality $d^{*}\left(g_{i} x H_{n}, g x H_{n}\right) \leq d\left(g_{i} x, g x\right)$, which yields the desired conclusion since $d\left(g_{i} x, g x\right) \rightarrow 0$.

Now suppose that $f\left(g_{i}\right) \rightarrow f(g)$. We want $g_{i} \rightarrow g$. Fix $\varepsilon>0$. Take $n$ such that $\operatorname{diam}\left(H_{n}\right)<\frac{\varepsilon}{3}$. By assumption we have $d^{*}\left(g_{i} H_{n}, g H_{n}\right) \rightarrow 0$, which allows us to choose sequences $\left(u_{i}\right)_{i}$ and $\left(v_{i}\right)_{i}$ in $H_{n}$ such that $d\left(g_{i} u_{i}, g v_{i}\right) \rightarrow 0$. Take $i_{0}$ such that for $i \geq i_{0}, d\left(g_{i} u_{i}, g v_{i}\right)<\frac{\varepsilon}{3}$. Since

$$
\begin{aligned}
d\left(g_{i}, g\right) & \leq d\left(g_{i}, g_{i} u_{i}\right)+d\left(g_{i} u_{i}, g v_{i}\right)+d\left(g v_{i}, g\right) \\
& =d\left(1, u_{i}\right)+d\left(g_{i} u_{i}, g v_{i}\right)+d\left(v_{i}, 1\right),
\end{aligned}
$$

we get $d\left(g_{i}, g\right)<\varepsilon$, for $i \geq i_{0}$, and we are done.

2.3. Proof of Corollary 1.3. By Theorem 1.2, it suffices to show that the property from this theorem holds for $G / N$ if it holds for $G$. Let $\pi: G \rightarrow G / N$ be the quotient homomorphism. Let $U$ be an open neighborhood of 1 in $G / N$. Let $V$ be an open neighborhood of 1 in $G / N$ whose closure is contained in $U$. Pick $H<\pi^{-1}(V)$ a closed subgroup of $G$ with $N(H)$ open and with $G / H$ locally compact. Define

$$
H^{\prime}=\overline{\pi(H)}
$$


where the closure is taken in $G / N$. Obviously $H^{\prime}$ is a closed subgroup of $G / N$ contained in $U$. The normalizer of $H^{\prime}$ in $G / N$ contains $\pi(N(H))$ and therefore is open. The natural function

$$
G / H \rightarrow G / \pi^{-1}\left(H^{\prime}\right) \cong(G / N) / H^{\prime}
$$

is surjective, continuous, and open. Thus $(G / N) / H^{\prime}$ is locally compact.

\section{Proof of Theorem 1.1}

Theorem 1.1 follows from Theorem 1.2 and from Lemma 3.2 below. Therefore, proving this lemma is all that remains to be done.

The following notion comes from [7]. It will be used in the proof of Lemma 3.2 through an application of Theorem 3.1 below. Assume we have a measure preserving Boolean action of a Polish group $G$ on $\mathcal{B}(X) / \mu$. We say that a function $f \in L^{\infty}(\mu)$ is $G$-continuous if $f \circ g_{n}$ converges to $f$ in the $L^{\infty}$-norm whenever $g_{n}$ converges to the identity in $G$. (By $L^{\infty}(\mu)$ we understand the real-valued $L^{\infty}(\mu)$.)

Theorem 3.1. ([7, Theorem 2.2], see also [8, Theorem 1.7]) A measure preserving Boolean action of $G$ on $\mathcal{B}(X) / \mu$ admits a spatial model if and only if $G$-continuous functions are dense in $L^{2}(\mu)$.

We note that since $\operatorname{Aut}(\mu)$ is a subgroup of $\mathrm{O}\left(L^{2}(\mu)\right)$, each continuous homomorphism $G \rightarrow \operatorname{Aut}(\mu)$, i.e., Boolean action of $G$, gives rise to a continuous homomorphism $G \rightarrow \mathrm{O}\left(L^{2}(\mu)\right)$, i.e., a continuous representation of $G$. We call this representation the representation induced by the Boolean action.

Recall the definition of property $(*)$ from the beginning of Subsection 2.1.

Lemma 3.2. Let $G$ be a group with property $(*)$. Then every Boolean action of $G$ has a spatial model.

Proof. Fix a standard Borel space $(X, \mathcal{B}(X), \mu)$ with a Borel probability measure $\mu$ and fix a Boolean action of $G$. We aim to show that $G$-continuous functions are dense in $L^{2}(\mu)$, which will prove the lemma by Theorem 3.1. We consider the representation of $G$ induced by the Boolean action. The first step of the proof consisting of an application of a fixed point theorem 
is borrowed from the proof of [8, Theorem 2.3]. Fix $f_{0} \in L^{2}(\mu)$ and $\varepsilon>0$. Consider

$$
B\left(f_{0}, \varepsilon\right)=\left\{f \in L^{2}(\mu):\left\|f-f_{0}\right\|_{2} \leq \varepsilon\right\} .
$$

Let $U_{f_{0}} \subseteq G$ be an open neighborhood of 1 such that $U_{f_{0}} \cdot f_{0} \subseteq B\left(f_{0}, \varepsilon\right)$. Since $G$ has property (*), we can fix a closed subgroup $H<G$ such that $H \subseteq U_{f_{0}}, G / H$ is locally compact and $N(H)$ is open.

Since $L^{2}(\mu)$ is reflexive, by the Banach-Alaoglu theorem, the closed ball $B\left(f_{0}, \varepsilon\right)$ is weakly compact. Let

$$
D={\overline{\operatorname{conv}\left\{h \cdot f_{0}: h \in H\right\}}}^{w}
$$

be the weak closure of the convex hull of the $H$-orbit of $f_{0}$ in $L^{2}(\mu)$. The set $D$ is a weakly compact convex $H$-invariant subset of $B\left(f_{0}, \varepsilon\right)$. By the RyllNardzewski fixed point theorem, see [3, Chapter V, Theorem 10.8], there is $f_{1} \in D$ which is $H$-fixed. Without loss of generality, we can assume that $f_{1} \in L^{\infty}(\mu)$. (If $f_{1}$ is not bounded replace it by $f^{M}$ satisfying $f^{M}(x)=f_{1}(x)$ if $|f(x)|<M$ and $f^{M}(x)=M$ if $|f(x)| \geq M$, for some $M>0$ such that $f^{M} \in B\left(f_{0}, \varepsilon\right)$.)

Let $\mathcal{A}$ be the smallest closed sublattice of $L^{2}(\mu)$ (i.e. $\mathcal{A}$ is a linear space closed under max and min) containing $f_{1}$, constant functions, and closed under the action of $N(H)$.

Our goal is to show that $G$-continuous functions are dense in $\mathcal{A}$ in the $L^{2}$-norm. Note that this will finish the proof since then, in particular, we get some $G$-continuous function $f$ such that $\left\|f-f_{1}\right\|_{2}<\varepsilon$. Since $\left\|f_{0}-f_{1}\right\|_{2}<\varepsilon$, where $f_{0}$ is the function we fixed at the beginning, we get $\left\|f-f_{0}\right\|_{2}<2 \varepsilon$.

Let $Q$ be a dense, countable subgroup of $N(H)$. For $h . f_{1} \in L^{2}(\mu)$ with $h \in Q$, let $f_{1, h}: X \rightarrow \mathbb{R}$ be a Borel function that is a representative of $h . f_{1}$. Let $\mathcal{C}$ be the countable algebra of Borel subsets of $X$ generated by the preimages under $f_{1, h}, h \in Q$, of rational intervals of $\mathbb{R}$. Let $\mathcal{B} \subseteq \mathcal{B}(X)$ be the $\sigma$-algebra generated by $\mathcal{C}$.

Claim 1. For $h \in N(H)$ and $B \in \mathcal{B}$ we have

$$
h .[B]_{\mu} \in \mathcal{B} / \mu .
$$


Proof of Claim 1. The conclusion of the claim holds for all $h \in Q$ by the definition of $\mathcal{B}$. Now equip $\mathcal{B}(X) / \mu$ with the metric

$$
\operatorname{dist}\left(\left[B_{1}\right]_{\mu},\left[B_{2}\right]_{\mu}\right)=\mu\left(B_{1} \triangle B_{2}\right) .
$$

Note that since $\mathcal{B}$ is a $\sigma$-subalgebra of $\mathcal{B}(X), \mathcal{B} / \mu$ is a closed subset of $\mathcal{B}(X) / \mu$. The action of $N(H)$ on $\mathcal{B}(X) / \mu$ is continuous. Since $Q$ is dense in $N(H)$, the claim follows.

Define the function $\pi: X \rightarrow\{0,1\}^{\mathcal{C}}$ by

$$
\pi(x)(B)=1 \text { iff } x \in B
$$

for $B \in \mathcal{C}$. The function $\pi$ is Borel. Set

$$
X^{\prime}=\pi(X), \mu^{\prime}=\pi_{*}(\mu),
$$

where, as usual, $\pi_{*}(\mu)(B)=\mu\left(\pi^{-1}(B)\right)$ for $B \subseteq X^{\prime}$ Borel. Note that there is a standard Borel set $Y \subseteq X^{\prime}$ with $\mu^{\prime}(Y)=1$ in which case

$$
\mathcal{B}\left(X^{\prime}\right) / \mu^{\prime} \cong \mathcal{B}(Y) /\left(\mu^{\prime}\lceil\mathcal{B}(Y)) .\right.
$$

Thus, we can assume that $X^{\prime}$ itself is standard Borel. Note that $X^{\prime}$ comes equipped with the topology inherited from $\{0,1\}^{\mathcal{C}}$, which we will use.

The $\sigma$-algebra $\mathcal{B}$ consists precisely of preimages under $\pi$ of elements of $\mathcal{B}\left(X^{\prime}\right)$. Mapping $B \in \mathcal{B}$ to the unique $B^{\prime} \in \mathcal{B}\left(X^{\prime}\right)$ with $B=\pi^{-1}\left(B^{\prime}\right)$ induces a measure preserving isomorphism

$$
\pi_{*}: \mathcal{B} / \mu \rightarrow \mathcal{B}\left(X^{\prime}\right) / \mu^{\prime}
$$

By Claim 1, for each $B_{0} \in \mathcal{B}\left(X^{\prime}\right)$ and $h \in N(H)$ there exists $B_{1} \in \mathcal{B}\left(X^{\prime}\right)$ such that

$$
h .\left[\pi^{-1}\left(B_{0}\right)\right]_{\mu}=\left[\pi^{-1}\left(B_{1}\right)\right]_{\mu},
$$

which allows us to define

$$
h .\left[B_{0}\right]_{\mu^{\prime}}=\left[B_{1}\right]_{\mu^{\prime}} .
$$

It is now easy to check that this is a measure preserving Boolean action of $N(H)$ on $\mathcal{B}\left(X^{\prime}\right) / \mu^{\prime}$ and that for $h \in N(H)$

$$
\pi_{*}\left(h \cdot[B]_{\mu}\right)=h . \pi_{*}\left([B]_{\mu}\right) .
$$


The measure preserving isomorphism (11) induces in the natural way a lattice isomorphism

$$
\pi_{*}: L^{2}\left(\mu\lceil\mathcal{B}) \rightarrow L^{2}\left(\mu^{\prime}\right) .\right.
$$

This lattice isomorphism is also an isometry between $L^{2}\left(\mu\lceil\mathcal{B})\right.$ and $L^{2}\left(\mu^{\prime}\right)$ and, when restricted to $L^{\infty}(\mu \uparrow \mathcal{B})$, it is an isometry between $L^{\infty}(\mu \uparrow \mathcal{B})$ and $L^{\infty}\left(\mu^{\prime}\right)$. Furthermore, note that for the representation of $N(H)$ in $\mathrm{O}\left(L^{2}(\mu\lceil\mathcal{B}))\right.$ induced by its Boolean action on $\mathcal{B} / \mu$ given by Claim 1 and for the representation of $N(H)$ in $\mathrm{O}\left(L^{2}\left(\mathcal{B}\left(X^{\prime}\right)\right)\right)$ induced by the Boolean action given by (12), we have by (13)

$$
\pi_{*}(h . f)=h . \pi_{*}(f)
$$

for $h \in N(H)$ and $f \in L^{2}(\mu\lceil\mathcal{B})$.

We show that

$$
\mathcal{A} \subseteq L^{2}(\mu \uparrow \mathcal{B})
$$

Indeed, since $L^{2}\left(\mu\lceil\mathcal{B})\right.$ is a closed sublattice of $L^{2}(\mu)$, to see the above inclusion, it suffices to show that $k . f_{1} \in L^{2}(\mu\lceil\mathcal{B})$ for each $k \in N(H)$, for which it suffices to see that $k . f_{1}$ is $\mathcal{B}$-measurable. The latter statement is a consequence of Claim 1.

Now it follows from (15), that $\pi_{*}(f)$ is defined for each $f \in \mathcal{A}$. Set

$$
\mathcal{A}^{\prime}=\left\{\pi_{*}(f): f \in \mathcal{A}\right\} \subseteq L^{2}\left(\mu^{\prime}\right) .
$$

Since $\mathcal{A}$ is a closed sublattice of $L^{2}(\mu), \mathcal{A}^{\prime}$ is a closed sublattice of $L^{2}\left(\mu^{\prime}\right)$.

Claim 2. $\mathcal{A}^{\prime} \supseteq L^{\infty}\left(\mu^{\prime}\right)$.

Proof of Claim 2. Since $\mathcal{A}^{\prime}$ is a closed sublattice of $L^{2}\left(\mu^{\prime}\right)$, it is enough to show that for every $\varepsilon>0$ and $B \in \mathcal{B}^{\prime}$ there is $q \in \mathcal{A}^{\prime}$ such that $\left\|\chi_{B}-q\right\|_{2}<$ $2 \varepsilon$. Let $\varepsilon>0$ be given. Recall at this point the definitions of $Q, \mathcal{C}$, and $\pi$. It follows from these definitions that for $k \in Q, \pi_{*}\left(k . f_{1}\right) \in L^{2}\left(\mu^{\prime}\right)$ has a continuous representative. For the remainder of the proof of this claim, we think of each $\pi_{*}\left(k . f_{1}\right)$ as an actual continuous function from $X^{\prime}$ to $\mathbb{R}$. Let $L \subseteq X^{\prime}$ be compact with $\mu^{\prime}(L)>1-\varepsilon^{2}$. Let $C(L)$ stand for the lattice of all continuous functions from $L$ to $\mathbb{R}$ with the $L^{\infty}$-norm. By the definition of $\pi$, the sublattice of $C(L)$ generated by the constant functions and $\left\{\pi_{*}\left(k . f_{1}\right)\lceil L: k \in Q\}\right.$ separates points of $L$. By the Stone-Weierstrass 
theorem for lattices, see [1, Chapter 2, Theorem 11.3], this sublattice is dense in $C(L)$. This fact allows us to pick $q \in \mathcal{A}^{\prime}$ with $\|\left(q\lceil L)-\chi_{B \cap L} \|_{2}<\varepsilon\right.$. Since $\mathcal{A}^{\prime}$ is a lattice with $0,1 \in \mathcal{A}^{\prime}$, we can assume that $0 \leq q \leq 1$. Now, by the choice of $L$, we have $\left\|q-\chi_{B}\right\|_{2}<2 \varepsilon$.

Set

$$
G_{0}=N(H) / H
$$

We will need the claim below to define a Boolean action of $G_{0}$ on $\mathcal{B}\left(X^{\prime}\right) / \mu^{\prime}$.

Claim 3. For $g_{1}, g_{2} \in N(H)$, if $g_{1}, g_{2}$ are in the same coset of $H$, then $g_{1} \cdot\left[B^{\prime}\right]_{\mu^{\prime}}=g_{2} \cdot\left[B^{\prime}\right]_{\mu^{\prime}}$ for each $B^{\prime} \in \mathcal{B}\left(X^{\prime}\right)$.

Proof of Claim 3. If $g_{1}, g_{2} \in N(H)$ are in the same coset of $H$ and $k \in$ $N(H)$, then $g_{1} k$ and $g_{2} k$ are in the same coset of $k^{-1} H k=H$. Thus,

$$
\left(\left(g_{2} k\right)^{-1} g_{1} k\right) \cdot f_{1}=f_{1},
$$

hence

$$
g_{1} \cdot\left(k \cdot f_{1}\right)=g_{2} \cdot\left(k \cdot f_{1}\right) .
$$

It follows from the definition of $\mathcal{B}$ that for each $B \in \mathcal{B}$ we have $g_{1} \cdot[B]_{\mu}=$ $g_{2} \cdot[B]_{\mu}$. Now from this equality, by (13), we have for $B \in \mathcal{B}$

$$
g_{1} \cdot \pi_{*}\left([B]_{\mu}\right)=\pi_{*}\left(g_{1} \cdot[B]_{\mu}\right)=\pi_{*}\left(g_{2} \cdot[B]_{\mu}\right)=g_{2} \cdot \pi_{*}\left([B]_{\mu}\right),
$$

and the conclusion of the claim follows.

Now Claim 3 allows us to define a measure preserving Boolean action of $G_{0}$ on $\mathcal{B}\left(X^{\prime}\right) / \mu^{\prime}$ by letting for $g H \in G_{0}$ and $B \in \mathcal{B}\left(X^{\prime}\right)$

$$
(g H) \cdot[B]_{\mu^{\prime}}=g \cdot[B]_{\mu^{\prime}} .
$$

We consider the representation of $G_{0}$ induced by the above Boolean action. Since $G_{0}$ is locally compact, by a combination of the theorem of Mackey [9] and Theorem 3.1, $G_{0}$-continuous functions for the above representation are dense in $L^{2}\left(\mu^{\prime}\right)$. We will derive from it the conclusion that $G$-continuous functions are dense in $\mathcal{A}$ in the $L^{2}$-norm, which will finish the proof. First we note that if $f \in \mathcal{A}$ and $\pi_{*}(f)$ is $G_{0}$-continuous, then $f$ is $G$-continuous. Indeed, obviously $\pi_{*}(f)$ is $N(H)$-continuous. By the fact that $\pi_{*}$ preserves the $L^{\infty}$-norm and by (14), it follows that $f$ is $N(H)$-continuous. Since $N(H)$ 
is open in $G$, every $N(H)$-continuous function is also $G$-continuous; thus, $f$ is $G$-continuous.

Now since $\pi_{*}(\mathcal{A})=\mathcal{A}^{\prime}$ and $\pi_{*}$ preserves the $L^{2}$-norm, we will be done if we show that $G_{0}$-continuous functions are dense in $\mathcal{A}^{\prime}$. But this follows from Claim 2 and the fact that $G_{0}$-continuous functions are dense in $L^{2}\left(\mu^{\prime}\right)$.

\section{REFERENCES}

[1] C.D. Aliprantis, O. Burkinshaw, Principles of Real Analysis, Academic Press, 1998.

[2] H. Becker, A Boolean G-space without a point realization, manuscript, December 2001.

[3] J.B. Conway, A Course in Functional Analysis, Springer-Verlag, 1990.

[4] A.I. Danilenko, Point realization of Boolean actions of countable inductive limits of locally compact groups, Mat. Fiz. Anal. Geom. 7 (2000), 35-48.

[5] S. Gao, Invariant Descriptive Set Theory, CRC Press, 2009.

[6] S. Gao, A.S. Kechris, On the classification of Polish metric spaces up to isometry, Mem. Amer. Math. Soc. 161 (2003), no. 766.

[7] E. Glasner, B. Tsirelson, B. Weiss, The automorphism group of the Gaussian measure cannot act pointwise, Israel J. Math. 148 (2005), 305-329.

[8] E. Glasner, B. Weiss, Spatial and non-spatial actions of Polish groups, Ergodic Theory Dynam. Systems 25 (2005), 1521-1538.

[9] G.W. Mackey, Point realisations of transformation groups, Illinois J. Math. 6 (1962), $327-335$.

[10] M. Malicki, S. Solecki, Isometry groups of separable metric spaces, Math. Proc. Cambridge Philos. Soc. 146 (2009), 67-81.

[11] J. Melleray, Compact metrizable groups are isometry groups of compact metric spaces, Proc. Amer. Math. Soc. 136 (2008), 1451-1455.

[12] D. Montgomery, L. Zippin, Topological Transformation Groups, Interscience Publishers, 1955.

[13] V.V. Uspenskij, On the group of isometries of the Urysohn universal metric space, Comment. Math. Univ. Carolin. 31 (1990), 181-182.

[14] F.W. Warner, Foundations of Differentiable Manifolds and Lie Groups, SpringerVerlag, 1983. 